\title{
Arbor
}

\section{Análisis de la presencia institucional del CSIC en el Web}

\section{Isidro Aguillo Caño y Begoña Granadino Goenechea}

Arbor CLXXIX, 705 (Septiembre 2004), 111-139 pp.

Este trabajo tiene como objetivo el estudio de la presencia del CSIC en el Web. Los indicadores cibermétricos utilizados, se basan en la estima del volumen de contenidos de los diferentes subdominios institucionales del CSIC y la cuantificación de la naturaleza hipertextual de las páginas Web de dichos subdominios.

Las áreas de Biología y Biomedicina, Recursos Naturales y Ciencia y Tecnología Físicas, son las áreas con mayor tamaño y visibilidad del Web. Ciencias Agrarias y Ciencia y Tecnología de Alimentos, son las áreas de menor tamaño y visibilidad del Web. El CSIC aparece en la posición 6.857 de popularidad según el Ranking Alexa, siendo la página Web principal del CSIC y la página Web del CINDOC las de mayor contribución.

\section{Introducción}

Los estudios sobre ciencia y tecnología empiezan a tener en cuenta la presencia de las instituciones académicas y de Investigación en el Web (Aguillo, 1998; 2000; Almind \& Ingwersen, 1997; Larson, 1996; Rousseau, 1997; Thellwall \& Harries, 2003).

La Cibermetría es una disciplina nueva (Bjorneborn, 2004; Bjorneborn \& Ingwersen, 2001; Thelwall et al., 2005; Ingwersen \& Bjorneborn, 2004) que intenta aplicar técnicas cienciométricas y bibliométricas al 
proceso de comunicación científica que tiene lugar en Internet. Este me dio ha resultado ser una excelente plataforma para la publicación y difusión del conocimiento científico generado por las distintas instituciones académicas y de investigación.

Este trabajo tiene como objetivo el estudio de la presencia de los centros del CSIC en el Web. El Web puede ser un buen indicador para medir visibilidad e impacto de las sedes de investigación y en este caso de los distintos centros del CSIC

En este estudio hemos analizado la presencia del CSIC en el Web utilizando como indicadores (Ingwersen, 1998; Li et al., 2003; Thelwall \& Harries, 2003; Vaughan \& Thelwall, 2003) el tamaño (número de páginas html/ centro) y la visibilidad (número de enlaces externos que recibe cada centro) de los centros que componen las ocho áreas científico-técnicas de esta institución (www.csic.es).

1. Humanidades y Ciencias Sociales: 19 centros

2. Biología y Biomedicina: 20 centros

3. Recursos Naturales: 27 centros

4. Ciencias Agrarias: 14 centros

5. Ciencia y Tecnologías Físicas: 28 centros

6. Ciencia y Tecnología de Materiales: 10 centros

7. Ciencia y Tecnología de Alimentos: 8 centros

8. Ciencia y Tecnologías Químicas: 14 centros

FIGURA 1

\section{NÚMERO DE CENTROS POR ÁREA}

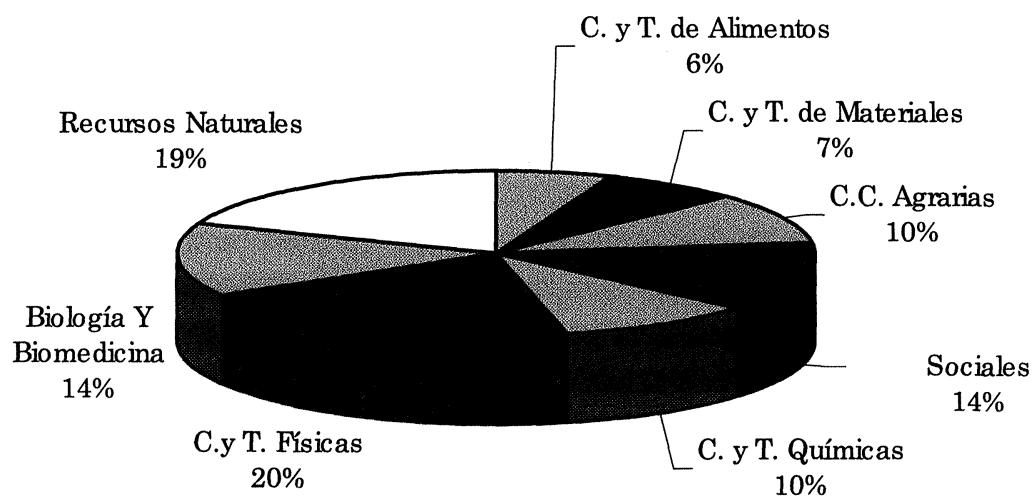




\section{Metodología}

Los indicadores cibermétricos utilizados en este estudio ya han sido descritos con anterioridad (EICSTES, 2002) y se basan fundamentalmente en la estima del volumen de contenidos de los diferentes subdominios institucionales del CSIC y la cuantificación de la naturaleza hipertextual de las páginas Web incluidas en dichos subdominios.

En primer lugar se identificó la URL de cada Centro, Instituto o unidad relevante del CSIC a partir del directorio publicado por el propio organismo en su sede web, complementado con datos actualizados obtenidos por navegación guiada las escasas ocasiones que no existían referencias concretas.

En general ha sido posible adjudicar un subdominio específico a cada unidad, aunque no todas ellas están bajo el dominio institucional genérico csic.es.

Los datos han sido extraídos con ayuda de los cinco motores de búsqueda que permiten la utilización de delimitadores: Google, Alltheweb, Altavista, MSN Search y Teoma. Para cada uno de los subdominios se ha calculado su tamaño, utilizando el delimitador site, excepto para Altavista (host) y Teoma:y MSNSearch (búsqueda avanzada). Los datos fueron recabados en dos momentos separados unos 6 meses (18 de julio y 23 de diciembre de 2003 respectivamente).

Con ayuda de Google se obtuvo el número y distribución de los ficheros ricos para cada subdominio (Aguillo,2002), mientras que para la distribución de contenidos por idioma se utilizó el motor Alltheweb.

La visibilidad fue calculada tanto en valor absoluto (comando link en Alltheweb) como relativo (utilizando el PageRank de Google en búsquedas neutras).

Por último la popularidad pudo ser recabada del motor de Alexa, que intercepta visitas a las diferentes sedes y genera listados ordenados sin indicar los valores absolutos.

\section{Resultados}

Datos generales del tamaño del Web de las áreas científicas del CSIC

Utilizando métodos indirectos de cibermetría, a través de motores de búsqueda, hemos determinado el tamaño en el Web de los distintos centros del CSIC que componen las ocho áreas científico-técnicas. La contri- 
bución relativa de cada centro al tamaño total del web de cada área científico-técnica es muy variable. Por ello analizamos inicialmente el tamaño del Web por área y posteriormente el tamaño de cada centro del CSIC.

El tamaño del Web de cada área científico-técnica está representado en la figura 1.

De las ocho áreas científico técnicas, la que tiene un mayor número de páginas Web es el área de Recursos Naturales, siendo ésta el área del CSIC que cuenta con mayor número de centros (27). El área de Biología y Biomedicina cuenta con 20 centros y tiene 42.332 páginas web. El área de Ciencia y Tecnologías Físicas con 28 centros, tiene 34.783 páginas, Ciencia y Tecnologías Químicas con 14 centros, 34.046 páginas, Ciencias Agrarias tiene 14 centros y un total de 8.514 páginas y Ciencia y Tecnología de Materiales con 10 centros , 7.583 páginas web. El área de Ciencia y Tecnología de alimentos, es el área del CSIC que tiene un menor numero de páginas, y es el área con menor numero de centros (7).

FIgURA 2

TAMAÑO DEL WEB

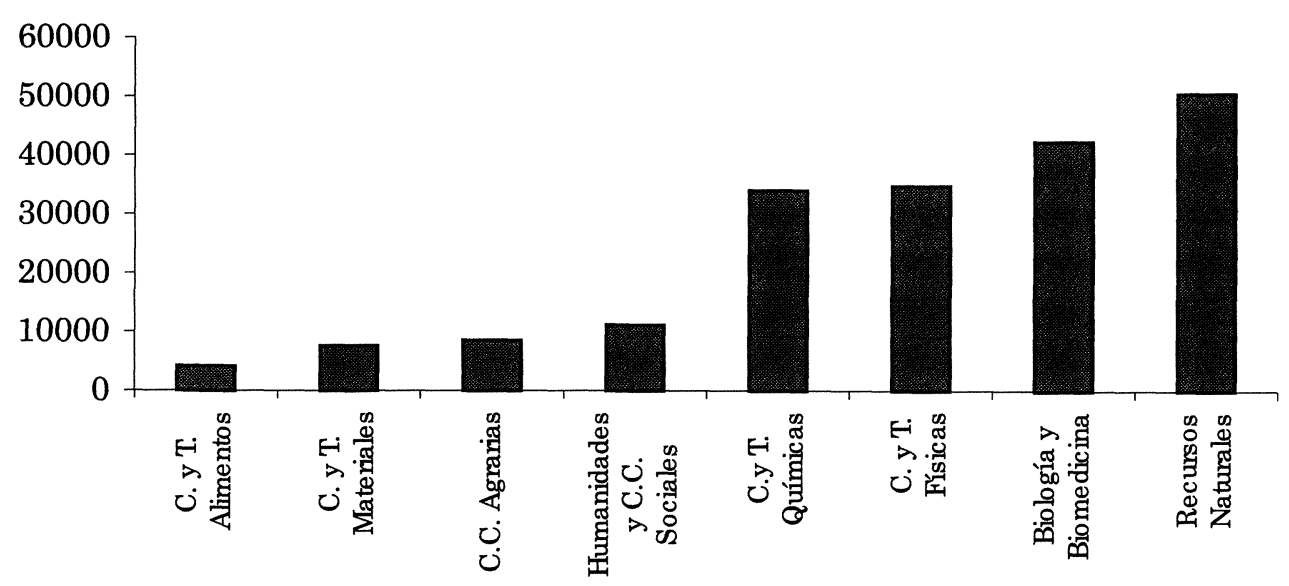

Tamaño del Web por centros

La contribución relativa de cada centro al tamaño del Web de cada área del CSIC es muy variable. En el área de Humanidades y Sociales 


\section{Análisis de la presencia institucional del CSIC en el Web}

formada por 19 centros, el CINDOC es el centro con mayor números de páginas Web y contribuye al tamaño total de esta área en un 56,8 \% . Otros centros tienen una contribución muy pequeña al número total de páginas del área como es el caso del Instituto Histórico de Hoffmeyer y el Instituto de Estudios Islámicos y de oriente Próximo.

TABLA 1. Humanidades y Ciencias Sociales

\begin{tabular}{|l|c|c|}
\hline \multicolumn{1}{|c|}{ CENTRO DEL CSIC } & DOMINIO & $\begin{array}{c}\text { TAMANO DEL } \\
\text { WEB }\end{array}$ \\
\hline Instituto Histórico Hoffmeyer (HH) & ih.csic.es/presentacion/hoffmeyer & 4 \\
\hline $\begin{array}{l}\text { Instituto de Estudios Islámicos y de Oriente } \\
\text { Próximo }\end{array}$ & ieiop.com & 27 \\
\hline Instituto de Arqueología de Mérida & iam.csic.es & 31 \\
\hline $\begin{array}{l}\text { Instituto de Estudios Sociales de Andalucía } \\
\text { IESA) }\end{array}$ & iesaa.csic.es & 34 \\
\hline $\begin{array}{l}\text { Instituto de Estudios Gallegos Padre } \\
\text { Sarmiento (IEGPS) }\end{array}$ & iegps.csic.es \\
\hline $\begin{array}{l}\text { Escuela Española de Historia y Arqueología en } \\
\text { Roma (EEHAR) }\end{array}$ & csic.it & 47 \\
\hline Escuela de estudios Hispano-Americanos & eeha.csic.es & 77 \\
\hline Instituto de la Lengua Española & ile.csic.es & 78 \\
\hline $\begin{array}{l}\text { Instituto de Historia de la Ciencia y } \\
\text { Documentación López Piñero }\end{array}$ & uv.es/ fresquet/TEXTOS & 128 \\
\hline Institución Milá y Fontanals & imf.csic.es & 160 \\
\hline Escuela de Estudios Árabes & eea.csic.es & 204 \\
\hline Instituto de Análisis Económico & iae-csic.uab.es & 239 \\
\hline Unidad de Políticas Comparadas (UPC) & iesam.csic.es & 241 \\
\hline $\begin{array}{l}\text { Instituto de la Gestión de la Innovación y del } \\
\text { Conocimiento (INGENIO) }\end{array}$ & ingenio.upv.es & 6.330 \\
\hline Instituto de Historia & ceh.csic.es & 632 \\
\hline Instituto de Filosofía (IFS) & ifs.csic.es & 763 \\
\hline Instituto de Filología & filol.csic.es & 1.010 \\
\hline Instituto de Economía y Geografía & cindoc.csic.es & 1.030 \\
\hline $\begin{array}{l}\text { Centro de Información y Documentación } \\
\text { Científica (CINDOC) }\end{array}$ & & \\
\hline
\end{tabular}

De los 20 centros que forman el área de Biología y Biomedicina, el Centro Nacional de Biotecnología es el que cuenta con mayor número de páginas Web, y contribuye con un $76,7 \%$ del total de páginas de esta área. $3 / 4$ de estas páginas se deben a una sección del grupo de trabajo de proteínas de este centro (pdg.cnb.uam.es), con bases de datos, y páginas personales de diferentes investigadores.

Por otra parte en el momento en el que se hizo el estudio, tanto el Instituto de Biología Molecular y Celular del Cáncer como el Instituto de Biología y Genética Molecular de Valladolid, contaban con un número nulo de páginas Web. Este se debe a que ambas páginas Web se encon- 


\section{Isidro Aguillo Caño y Begoña Granadino Goenechea}

traban en proceso de cambio, ya que en el momento actual y con la nue va dirección Web, ambos centros contribuyen con un número mayor de páginas Web al tamaño total del área. El IBMCC (www.cicancer.org) con 3710 páginas, y el IBGM de Valladolid (www.ibgm.med.uva.es) con 1680 .

TABLA 2. Biología y Biomedicina

\begin{tabular}{|c|c|c|}
\hline CENTRO DEL CSIC & DOMINIO & TAMAÑO DEL WEB \\
\hline $\begin{array}{l}\text { Instituto de Biología Molecular y Celular del } \\
\text { Cáncer (IBMCC) }\end{array}$ & www-cicancer.usal.es & 0 \\
\hline $\begin{array}{l}\text { Instituto de Biología y Genética Molecular, } \\
\text { Valladolid }\end{array}$ & ibgmva.csic.es & 8 \\
\hline Instituto de Biología Molecular de Barcelona & ibmb.csic.es & 21 \\
\hline $\begin{array}{l}\text { Instituto de Farmacología y Toxicología } \\
\text { (IFT) }\end{array}$ & ift.csic.es & 29 \\
\hline Instituto de Bioquímica (IB) & ib.csic.es & 46 \\
\hline $\begin{array}{l}\text { Instituto de Bioquímica Vegetal y } \\
\text { Fotosíntesis (IBVF) }\end{array}$ & ibvf.cartuja.csic.es & 53 \\
\hline Unidad de Biofísica & ehu.es/biofisica & 59 \\
\hline Instituto de Biomedicina de Valencia (IBV) & ibv.csic.es & 82 \\
\hline Instituto de Neurociencias (IN) & in.umh.es & 85 \\
\hline $\begin{array}{l}\text { Instituto de Parasitología Y Biomedicina } \\
\text { López Neyra }\end{array}$ & ipb.csic.es & 161 \\
\hline Instituto de Microbiología Bioquímica & imb.usal.es & 213 \\
\hline $\begin{array}{l}\text { Instituto de Investigaciones Biomédicas de } \\
\text { Barcelona (IIBB) }\end{array}$ & iibb.csic.es & 224 \\
\hline $\begin{array}{l}\text { Centro de Investigaciones Científicas Isla de } \\
\text { la Cartuja (CIC) }\end{array}$ & cartuja.csic.es & 244 \\
\hline Instituto de Neurobiología Ramón y Cajal & cajal.csic.es & 521 \\
\hline $\begin{array}{l}\text { Instituto de Biología Molecular y Celular de } \\
\text { plantas'E Primo Yufera' }\end{array}$ & ibmcp.upv.es & 667 \\
\hline Centro de Investigación y Desarrollo (CID) & cid.csic.es & 709 \\
\hline $\begin{array}{l}\text { Centro de Biología Molecular Severo Ochoa } \\
\text { (CBM) }\end{array}$ & cbm.uam.es & 1.780 \\
\hline Centro de Investigaciones Biológicas (CIB) & cib.csic.es & 1.780 \\
\hline $\begin{array}{l}\text { Instituto de Investigaciones Biomédicas } \\
\text { Alberto Sols (IIB) }\end{array}$ & iib.uam.es & 3.150 \\
\hline Centro Nacional de Biotecnología & cnb.uam.es & 32.500 \\
\hline
\end{tabular}

El área de Recursos Naturales cuenta con 27 centros con sede Web propia. El Museo de Ciencias Naturales aporta un 33\% de las páginas y el IMEDEA un 15,8\%. El Centro Mediterráneo de Investigaciones Marinas y Medioambientales tiene un dominio propio y contiene páginas Web del ICM y del UTM. 


\section{Análisis de la presencia institucional del CSIC en el Web}

TABLA 3. Recursos Naturales

\begin{tabular}{|c|c|c|}
\hline CENTRO DEL CSIC & DOMINIO & $\begin{array}{l}\text { TAMAÑO DEL } \\
\text { WEB }\end{array}$ \\
\hline Instituto Andaluz de Ciencias de la Tierra (IACT) & ugr.es/ offiact/ & 8 \\
\hline $\begin{array}{c}\text { Instituto de Recursos Naturales y Agrobiología de } \\
\text { Salamanca (IRNASA) }\end{array}$ & irnasa.csic.es & 24 \\
\hline Centro de Investigación sobre desertificación & uv.es/cide/ & 32 \\
\hline Instituto Botánico de Barcelona & institutbotanic.bcn.es & 45 \\
\hline $\begin{array}{l}\text { Centro de Edafología y Biología aplicada del Segura } \\
\text { (CEBAS) }\end{array}$ & cebas.csic.es & 54 \\
\hline Instituto de Astronomía y Geodesia (IAG) & mat.ucm.es/deptos/iag & 72 \\
\hline Instituto de Productos Naturales y Agrobiología (IPNA) & ipna.csic.es & 76 \\
\hline Instituto de Acuicultura de Torre de la Sal & iats.csic.es & 89 \\
\hline Instituto de Geología Económica (IGE) & ige.csic.es & 111 \\
\hline Instituto de Ciencias Marinas de Andalucía (ICMAN) & icman.csic.es & 112 \\
\hline $\begin{array}{l}\text { Instituto de Investigación en Recursos Cinegéticos } \\
\text { (IREC) }\end{array}$ & uclm.es/irec & 129 \\
\hline Instituto Pirenaico de Ecología (IPE) & ipe.csic.es & 180 \\
\hline Estación Experimental de Zonas Áridas (EEZA) & eeza.csic.es & 243 \\
\hline Centro de Ciencias medioambientales (CCMA) & ccma.csic.es & 501 \\
\hline Instituto de Agroquímica y Tecnología de los Alimentos & iata.csic.es & 594 \\
\hline Real Jardín Botánico (RJB) & rjb.csic.es & 717 \\
\hline Centro de Estudios Avanzados de Blanes (CEAB) & ceab.csic.es & 902 \\
\hline Instituto de Investigaciones Marinas & iim.csic.es & 1.160 \\
\hline Instituto de Ciencias de la Tierra Jaume Almera & ija.csic.es & 1.360 \\
\hline $\begin{array}{l}\text { Instituto de Recursos Naturales y Agrobiología de Sevilla } \\
\text { (IRNAS) }\end{array}$ & irnase.csic.es & 2.130 \\
\hline Estación Experimental del Zaidín & eez.csic.es & 2.280 \\
\hline Instituto de Ciencias del Mar & icm.csic.es & 2.770 \\
\hline Unidad de Tecnología Marina (UTM) & utm.csic.es & 3.230 \\
\hline Estación Biológica de Doñana & ebd.csic.es & 3.640 \\
\hline $\begin{array}{c}\text { Centro Mediterráneo de investigaciones marinas y } \\
\text { medioambientales (UTM+ ICM) }\end{array}$ & cmima.csic.es & 5.300 \\
\hline $\begin{array}{l}\text { Instituto Mediterráneo de Estudios Avanzados } \\
\text { (IMEDEA) }\end{array}$ & imedea.uib.es & 7.970 \\
\hline Museo Nacional de Ciencias Naturales (MNCN) & mncn.csic.es & 16.700 \\
\hline
\end{tabular}

En el área de Ciencias Agrarias hay 14 centros con páginas Web. La EEZ contribuye con un $52 \%$ al número total de páginas de esta área. El Centro de menor contribución es el IARN que en el momento del análisis, contaba solo con 1 pagina Web desarrollada.

El área de Ciencia y Tecnologías Físicas comprende 28 centros con direcciones Web propias. De ellos el IFIC y el IMEDEA son los que tienen mayor número de páginas y contribuyen con un $40 \%$ y un $23 \%$ respectivamente, al número total de páginas Web de esta área. Otros Centros como el Instituto de Microelectrónica de Sevilla y el Instituto de Física aplicada, tienen muy pocas páginas Web en sus dominios (imse.cnm.es y ifa.csic.es), teniendo la mayoría de sus páginas en las SUPERsedes del CETEF (cetef.csic.es) y del CNM (cnm.es) respectivamente. El Centro de 
Tecnologías Físicas Leonardo Torres Quevedo (CETEF) incluye además del Instituto de Física Aplicada (IFA), el Instituto de Acústica y el Instituto de Automática Industrial. El Centro de Física Miguel A. Catalán (CFMAC) incluye Instituto de Estructura de la Materia (IEM) y el Instituto de Matemática y Física Fundamental (IMAFF) y el Instituto de Óptica Daza de Valdés. El IMAFF a su vez tiene 2 direcciones Web propias diferentes: imaff.cfmac.csic y imaff.csic.es. El Centro Nacional de Microelectrónica (CNM) comprende el Instituto de Microelectrónica de Sevilla, el Instituto de Microelectrónica de Barcelona (IMB-CNM) y el Instituto de Microelectrónica de Madrid.

TABLA 4. Ciencias Agrarias

\begin{tabular}{|c|c|c|}
\hline CENTRO DEL CSIC & DOMINIO & $\begin{array}{l}\text { TAMAÑO DEL } \\
\text { WEB }\end{array}$ \\
\hline $\begin{array}{l}\text { Instituto de Agrobiotecnología y Recursos } \\
\text { Naturales (IARN), Navarra }\end{array}$ & unavarra.es/invest/biotec.htm & 1 \\
\hline $\begin{array}{l}\text { Instituto de productos Naturales y Agrobiología } \\
\text { (IPNA), Tenerife }\end{array}$ & ipna.csic.es & 4 \\
\hline $\begin{array}{l}\text { Instituto de recursos Naturales y Agrobiología } \\
\text { de Salamanca (IRNASA) }\end{array}$ & irnasa.csic.es & 18 \\
\hline Misión Biológica de Galicia (MBG) & mbg.csic.es & 24 \\
\hline $\begin{array}{l}\text { Instituto de Investigaciones Agrobiológicas de } \\
\text { Galicia (IIAG) }\end{array}$ & iiag.csic.es & 25 \\
\hline $\begin{array}{l}\text { Centro de Edafología y Biología Aplicada del } \\
\text { Segura (CEBAS), Murcia }\end{array}$ & cebas.csic.es & 39 \\
\hline Estación Agrícola Experimental (EAE), León & eae.csic.es & 49 \\
\hline $\begin{array}{l}\text { Estación Experimental La Mayora (EELM), } \\
\text { Málaga }\end{array}$ & eelm.csic.es & 129 \\
\hline $\begin{array}{l}\text { Instituto de Agroquímica y Tecnología de } \\
\text { Alimentos (IATA), Valencia }\end{array}$ & iata.csic.es & 409 \\
\hline $\begin{array}{l}\text { Centro de Ciencias Medioambientales (CCMA), } \\
\text { Madrid }\end{array}$ & ccma.csic.es & 518 \\
\hline $\begin{array}{l}\text { Instituto de Agricultura Sostenible (IAS), } \\
\text { Córdoba }\end{array}$ & ias.csic.es & 778 \\
\hline $\begin{array}{l}\text { Estación Experimental de Aula Dei (EEAD), } \\
\text { Zaragoza }\end{array}$ & eead.csic.es & 791 \\
\hline $\begin{array}{l}\text { Instituto de recursos Naturales y Agrobiología } \\
\text { de Sevilla (IRNAS) }\end{array}$ & irnase.csic.es & 1291 \\
\hline $\begin{array}{l}\text { Estación Experimental del Zaidín (EEZ), } \\
\text { Granada }\end{array}$ & eez.csic.es & 4438 \\
\hline
\end{tabular}




\section{Análisis de la presencia institucional del CSIC en el Web}

TABla 5. Ciencia y Tecnologías Físicas

\begin{tabular}{|c|c|c|}
\hline $\begin{array}{c}\text { CENTRO DEL CSIC } \\
\end{array}$ & DOMINIO & TAMAÑO DEL WEB \\
\hline Instituto de Microelectrónica de Sevilla & imse.cnm.es & 2 \\
\hline Instituto de Física Aplicada (IFA) & ifa.csic.es & 5 \\
\hline Observatorio de Física Cósmica del Ebro (OE) & obsebre.es & 10 \\
\hline $\begin{array}{l}\text { Laboratorio de Física de Sistemas Pequeños y } \\
\text { Nanotecnología }\end{array}$ & fsp.csic.es & 17 \\
\hline Instituto de Astronomía y Geodesia (IAG) & mat.ucm.es/deptos/iag & 72 \\
\hline Centro Nacional de Aceleradores (CNA) & us.es/cna & 107 \\
\hline Instituto de Öptica Daza de Valdés & io.cfmac.csic.es & 143 \\
\hline $\begin{array}{l}\text { Instituto de Microelectrónica de Barcelona (IMB- } \\
\text { CNM) }\end{array}$ & cnm.es/imb & 199 \\
\hline Instituto de Microelectrónica de Madrid & imm.cnm.csic.es & 239 \\
\hline Instituto de Estructura de la Materia (IEM) & iem.cfmac.csic.es & 316 \\
\hline $\begin{array}{l}\text { Instituto de Matemática y Física Fundamental } \\
\text { (IMAFF) }\end{array}$ & imaff.cfmac.csic.es & $158+199$ * \\
\hline Centro Técnico de Informática (CTI) & cti.csic.es & 430 \\
\hline Instituto de Física Teórica & gesalerico.ft.uam.es & 514 \\
\hline Centro de Astrobiología (CAB) & cab.inta.es & 658 \\
\hline Instituto de Estudios Espaciales de Cataluña & ieec.fcr.es & 759 \\
\hline Instituto de Robótica e Informática (IRII) & www-iri.upc.es & 765 \\
\hline Instituto de Astrofísica de Andalucía & iaa.es & 927 \\
\hline Instituto de Acústica & ia.csic.es & 1.100 \\
\hline Instituto de Automática Industrial & iai.csic.es & 1.420 \\
\hline $\begin{array}{l}\text { Instituto de Investigación en Inteligencia } \\
\text { artificial (IIIA) }\end{array}$ & iiia.csic.es & 1.690 \\
\hline Instituto de Física de Cantabria (IFCA) & ifca.unican.es & 1.720 \\
\hline $\begin{array}{l}\text { Instituto Mediterráneo de Estudios Avanzados } \\
\text { (IMEDEA) }\end{array}$ & www-iri.upc.es & 7.970 \\
\hline Instituto de Física Corpuscular (IFIC) & ific.uv.es & 14.000 \\
\hline \multicolumn{3}{|l|}{ SUPERSEDES } \\
\hline $\begin{array}{l}\text { Centro de Tecnologías Físicas Leonardo Torres } \\
\text { Quevedo (CETEF) }\end{array}$ & cetef.csic.es & 6 \\
\hline Centro de Física Miguel A. Catalán (CFMAC) & cfmac.csic.es & 635 \\
\hline Centro nacional de Microelectrónica (CNM) & cnm.es & 722 \\
\hline
\end{tabular}

El área de Tecnología de Alimentos está formada por 8 centros, siendo el IIM y el IG los que tienen una contribución mayor al número total de páginas Web del área. El IIM contribuye con un $38 \%$ de páginas y el IG con un $45,7 \%$.

Sin embargo el Instituto de Nutrición y Bromatología no tiene página Web propia desarrollada. 


\section{Isidro Aguillo Caño y Begoña Granadino Goenechea}

TABLA 6. Tecnología de Alimentos

\begin{tabular}{|l|c|c|}
\hline \multicolumn{1}{|c|}{ CENTRO DEL CSIC } & DOMINIO & TAMAÑO DEL WEB \\
\hline $\begin{array}{l}\text { Instituto de Nutrición y Bromatología } \\
\text { (INB) }\end{array}$ & & 0 \\
\hline $\begin{array}{l}\text { Instituto de Fermentaciones } \\
\text { Industriales (IFI) }\end{array}$ & ifi.csic.es & 3 \\
\hline $\begin{array}{l}\text { Instituto de Productos Lácteos de } \\
\text { Asturias (IPLA) }\end{array}$ & ipla.csic.es & 12 \\
\hline $\begin{array}{l}\text { Centro de Edafología Y Biología } \\
\text { Aplicada del Segura (CEBAS) }\end{array}$ & cebas.csic.es & 39 \\
\hline Instituto del Frío (IF) & if.csic.es & 169 \\
\hline $\begin{array}{l}\text { Instituto de Agroquímica y Tecnología } \\
\text { de Alimentos (IATA) }\end{array}$ & iata.csic.es & 409 \\
\hline $\begin{array}{l}\text { Instituto de Investigaciones Marinas } \\
\text { (IIM) }\end{array}$ & iim.csic.es & 1.586 \\
\hline Instituto de La Grasa (IG) & ig.csic.es & 1.874 \\
\hline
\end{tabular}

En el área de Tecnología de Materiales hay 10 centros y los centros con mayor número de páginas son el ICMAB (32,8\%) y el ICMM (33,36\%). El ICMAB tiene 2 direcciones Web: icmab.es que tenía 2350 páginas en el momento en el que se hizo el estudio, y icmab.csic.es con 126 páginas.

TABLA 7. Tecnología de Materiales

\begin{tabular}{|l|c|c|}
\hline \multicolumn{1}{|c|}{ CENTRO DEL CSIC } & DOMINIO & TAMAÑO DEL WEB \\
\hline Instituto de Cerámica y vidrio (ICV) & icv.csic.es & 79 \\
\hline $\begin{array}{l}\text { Instituto de Ciencias Materiales de Aragón } \\
\text { (ICMA) }\end{array}$ & icma.csic.unizar.es & 89 \\
\hline $\begin{array}{l}\text { Instituto de Ciencias Materiales de Sevilla } \\
\text { (ICMS) }\end{array}$ & icmse.cartuja.csic.es & 122 \\
\hline $\begin{array}{l}\text { Centro Nacional de Investigaciones Metalúrgicas } \\
\text { (CENIM) }\end{array}$ & cenim.csic.es & 206 \\
\hline $\begin{array}{l}\text { Instituto de Ciencias de la Construcción Eduardo } \\
\text { Torroja (IETCC) }\end{array}$ & ietcc.csic.es & 208 \\
\hline $\begin{array}{l}\text { Instituto de Ciencia y Tecnología de polímeros } \\
\text { (ICTP) }\end{array}$ & ictp.csic.es & 219 \\
\hline $\begin{array}{l}\text { Centro de Investigaciones Científicas Isla } \\
\text { de la Cartuja (CIC) }\end{array}$ & cartuja.csic.es & 244 \\
\hline Unidad de Física de Materiales (UFM) & csic.sw.ehu.es & 1.410 \\
\hline $\begin{array}{l}\text { Instituto de Ciencias Materiales de Barcelona } \\
\text { (ICMAB) }\end{array}$ & icmab.es & $126+2.350$ \\
\hline $\begin{array}{l}\text { Instituto de Ciencias Materiales de Madrid } \\
\text { (ICMM) }\end{array}$ & icmm.csic.es & 2.530 \\
\hline
\end{tabular}

De los 14 centros del área de Ciencia y Tecnologías Químicas el IQFR es el centro con mayor número de páginas y supone un $90 \%$ del total de páginas Web de esta área. Este número de páginas tan alto se debe al elevado número de páginas Web del departamento de cristalografía, que incluye bases de datos de compuestos químicos. 


\section{Análisis de la presencia institucional del CSIC en el Web}

El Instituto de Química Orgánica General tiene dos direcciones web diferentes: iqo.csic.es y iqog.csic.es. Este centro pertenece al Centro de Química Orgánica Manuel Lora Tamayo (cnqo.csic.es), que en el momento del estudio tenía 11 páginas Web. El Instituto de Química Médica también pertenece a este centro.

TABLA 8. Ciencia y Tecnologías Químicas

\begin{tabular}{|c|c|c|}
\hline CENTRO DEL CSIC & DOMINIO & TAMAÑO DEL WEB \\
\hline $\begin{array}{l}\text { Instituto de Investigaciones } \\
\text { Químicas (IIQ) }\end{array}$ & iiq.cartuja.csic.es & 26 \\
\hline Instituto de Carboquímica & icb.csic.es & 42 \\
\hline $\begin{array}{l}\text { Laboratorio de Investigación en } \\
\text { Tecnologías de la Combustión } \\
\text { (LITEC) }\end{array}$ & litec.csic.es & 52 \\
\hline $\begin{array}{l}\text { Instituto de Tecnología Química } \\
\text { (ITQ) }\end{array}$ & upv.es/itq & 54 \\
\hline $\begin{array}{l}\text { Instituto de Productos Naturales y } \\
\text { Agrobiología (IPNA) }\end{array}$ & ipna.csic.es & 76 \\
\hline $\begin{array}{l}\text { Instituto de Ciencia de Materiales } \\
\text { de Aragón (ICMA) }\end{array}$ & icma.csic.unizar.es & 89 \\
\hline $\begin{array}{l}\text { Instituto de Química orgánica } \\
\text { general (IQOG) }\end{array}$ & iqo.csic.es & $114+45$ \\
\hline Instituto de Química Médica (IQM) & iqm.csic.es & 180 \\
\hline $\begin{array}{l}\text { Instituto Nacional del Carbón } \\
\text { (INCAR) }\end{array}$ & incar.csic.es & 220 \\
\hline $\begin{array}{l}\text { Centro de Investigaciones } \\
\text { Científicas Isla de la Cartuja } \\
\text { (CIC) }\end{array}$ & cartuja.csic.es & 244 \\
\hline $\begin{array}{l}\text { Instituto de Investigaciones } \\
\text { Químicas y Ambientales J.P.Vila } \\
\text { (IIQAB) }\end{array}$ & iiqab.csic.es & 394 \\
\hline $\begin{array}{l}\text { Centro de Investigación y } \\
\text { Desarrollo }\end{array}$ & cid.csic.es & 709 \\
\hline $\begin{array}{l}\text { Instituto de Catálisis y } \\
\text { Petroleoquímica }\end{array}$ & icp.csic.es & 1.090 \\
\hline $\begin{array}{l}\text { Instituto de Química Física } \\
\text { Rocasolano (IQFR) }\end{array}$ & iqfr.csic.es & 30.700 \\
\hline
\end{tabular}

De todos estos datos podemos determinar que los 4 centros del CSIC con mayor tamaño en el Web son:

- El Centro Nacional de Biotecnología (cnb.uam.es) centro mixto CSIC-UAM, del área de Biología y Biomedicina. 32.500 páginas Web.

- El Instituto de Química Física Rocasolano (iqfr.csic.es) centro propio del consejo del área de Ciencia y Tecnologías Químicas............30.700 páginas Web.

- EL Museo Nacional Ciencias Naturales (mncn.csic.es) centro propio del consejo del área de Recursos Naturales. 16.700 páginas Web. 


\section{Isidro Aguillo Caño y Begoña Granadino Goenechea}

- El Instituto de Física Corpuscular (ific.uv.es) centro mixto CSIC.UV, del área de Ciencia y Tecnologías Físicas. 14.000 páginas Web.

\section{Distribución del tamaño por CC.AA.}

La distribución del tamaño del Web de los distintos centros del CSIC, por Comunidades Autónomas se refleja en la tabla 12.

Madrid es la Comunidad Autónoma con mayor peso en la Web del CSIC, lo cual es lógico ya que es la Comunidad Autónoma con mayor numero de centros (40).

Cataluña tiene 18 centros del CSIC y contribuye con un $10,63 \%$ al peso de la Web del CSIC. Andalucía y Valencia con 20 y 10 centros respectivamente contribuyen con un $6 \%$ cada una al peso total del Web. En Baleares el IMEDEA, que es el único centro del CSIC de esta Comunidad Autónoma, contribuye con un 2,5\%. El resto de las Comunidades tienen una contribución pequeña al tamaño total del Web del CSIC. Cantabria, Castilla La Mancha, Canarias, Murcia y Navarra, cuentan con 1 único centro del CSIC. Extremadura, Asturias y el País Vasco tienen 2 centros del CSIC cada una. Galicia cuenta con 4 centros, Castilla León con 5 y Aragón con 6, todos ellos con un número bajo de páginas Web. La Rioja es la única Comunidad Autónoma que no cuenta con ningún centro del CSIC. Sin embargo existe un centro del CSIC en Roma (www.csic.it), que contribuye con un $0,02 \%$ al peso total del Web del CSIC.

TABLA 9

\begin{tabular}{|c|c|c|}
\hline COMUNIDADES & TAMAÑ & PESO \\
\hline Navarra & 1 & $0,00 \%$ \\
\hline Extremadura & 31 & $0,01 \%$ \\
\hline Italia & 54 & $0,02 \%$ \\
\hline Murcia & 54 & $0,02 \%$ \\
\hline Canarias & 76 & $0,02 \%$ \\
\hline Castilla La Mancha & 129 & $0.04 \%$ \\
\hline Asturias & 232 & $0,07 \%$ \\
\hline Castilla León & 320 & $0,10 \%$ \\
\hline País Vasco & 1.469 & $0,46 \%$ \\
\hline Galicia & 1.686 & $0,53 \%$ \\
\hline Cantabria & 1.721 & $0,54 \%$ \\
\hline Aragón & 1.828 & $0,57 \%$ \\
\hline Baleares & 7.970 & $2,50 \%$ \\
\hline Valencia & 21.093 & $6,62 \%$ \\
\hline Andalucía & 21.881 & $6,86 \%$ \\
\hline Cataluña & 33.896 & $10,63 \%$ \\
\hline Madrid & 226.309 & $71,00 \%$ \\
\hline
\end{tabular}


FiguRA 3

TAMAÑO POR COMUNIDADES AUTONOMAS

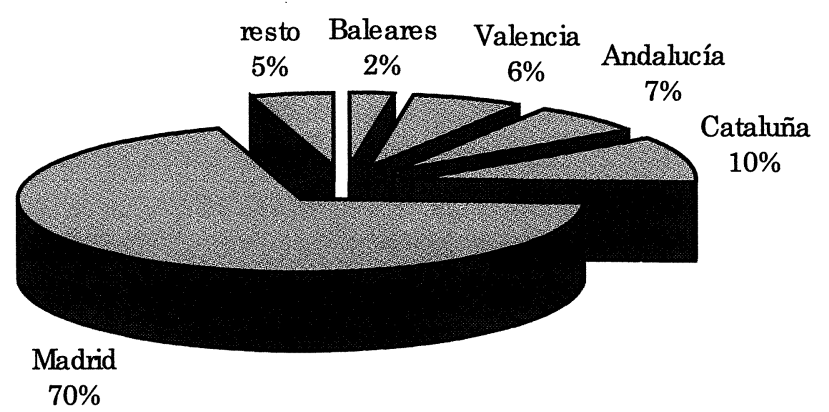

\section{Idioma de las páginas Web}

En todas las áreas el $90 \%$ de las paginas utilizan español o inglés como idioma en un porcentaje similar con la única excepción del área de Humanidades y Ciencias Sociales que utiliza el español en el $88 \%$ de sus páginas. Estos datos están en consonancia con el hecho de que el inglés sea la lengua franca de la comunicación científica. Inclusive en las áreas de Ciencia y Tecnología Físicas y Ciencia y Tecnología Químicas, predominan sobre el español las páginas en inglés (55,31 \% y 57 \% respectivamente).

El porcentaje de páginas en francés, alemán, portugués, italiano, catalán, gallego y eusquera es muy bajo, siendo inferior a un $10 \%$ en todas las áreas a excepción del área de CC Agrarias, en la que el Francés está en un $15 \%$ de las paginas, debido fundamentalmente a las páginas en este último idioma de la Estación Experimental del Zaidín. De estos idiomas, el francés y el catalán son los más utilizados. En el área de Recursos Naturales el francés ocupa un 4,47\% y el Catalán un 3,45\%. En Ciencia y Tecnologías Físicas el catalán esta en un 1,32 \% de las páginas, en Ciencia y Tecnología de Materiales en un $3 \%$ y en Ciencia y Tecnología Químicas en un 2,24. 
Isidro Aguillo Caño y Begoña Granadino Goenechea

TABLA 10

\begin{tabular}{|c|c|c|c|}
\hline AREA CIENTIFICA & $\begin{array}{c}\text { TAMAÑO } \\
\text { (Google) }\end{array}$ & $\begin{array}{c}\text { \% PAGINAS EN } \\
\text { ESPAÑOL }\end{array}$ & $\begin{array}{c}\text { \% PAGINAS } \\
\text { INGLES }\end{array}$ \\
\hline Ciencia y Tecnología de Alimentos & 4.092 & $63,02 \%$ & $32,13 \%$ \\
\hline Ciencia y Tecnología de Materiales & 7.583 & $50,44 \%$ & $41,98 \%$ \\
\hline Ciencias Agrarias & 8.514 & $47,58 \%$ & $37,42 \%$ \\
\hline Humanidades y CC Sociales & 11.136 & $88,53 \%$ & $4,51 \%$ \\
\hline Ciencia y Tecnologías Químicas & 34.046 & $40,7 \%$ & $57 \%$ \\
\hline Ciencia y Tecnologías Físicas & 34.783 & $38,21 \%$ & $55,31 \%$ \\
\hline Biología y Biomedicina & 42.332 & $64,36 \%$ & $35,17 \%$ \\
\hline Recursos Naturales & 50.429 & $56,64 \%$ & $35,03 \%$ \\
\hline
\end{tabular}

En la siguiente tabla se muestran las principales sedes multilingües. El Instituto de Química Física Rocasolano y el Instituto de Investigación en Inteligencia Artificial, son los centros del CSIC con mayor número de páginas Web en ingles. En la Estación Experimental del Zaidín, además de las páginas en inglés hay un alto número de páginas en francés. Hay que destacar también el alto porcentaje de páginas en Catalán del Instituto de Ciencias de la Tierra Jaume Almera.

TABLA 11

\begin{tabular}{|c|c|c|c|c|c|c|}
\hline Sedes & Dominio & Español & Inglés & Francés & Alemán & Catalán \\
\hline $\begin{array}{l}\text { Instituto de Química Física } \\
\text { Rocasolano }\end{array}$ & igfr.csic.es & 146 & 2109 & 0 & 1 & 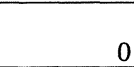 \\
\hline $\begin{array}{l}\text { Instituto de Investigación } \\
\text { en Inteligencia Artificial }\end{array}$ & iiia.csic.es & 235 & 2015 & 2 & 2 & 69 \\
\hline $\begin{array}{l}\text { Instituto de Ciencias del } \\
\text { Mar }\end{array}$ & icm.csic.es & 332 & 1750 & 6 & 14 & 88 \\
\hline $\begin{array}{l}\text { Centro Nacional de } \\
\text { Microelectrónica }\end{array}$ & cnm.es & 318 & 1443 & 0 & 0 & 44 \\
\hline $\begin{array}{l}\text { Instituto de } \\
\text { Microelectrónica de Sevilla }\end{array}$ & imse.cnm.es & 166 & 684 & 0 & 0 & 0 \\
\hline $\begin{array}{l}\text { Instituto de Automática } \\
\text { Industrial }\end{array}$ & iai.csic.es & 274 & 772 & 2 & 1 & 0 \\
\hline $\begin{array}{l}\text { Estación Experimental del } \\
\text { Zaidín }\end{array}$ & eez.csic.es & 1120 & 1776 & 1298 & 0 & 0 \\
\hline $\begin{array}{l}\text { Instituto de Ciencia de } \\
\text { Materiales de Barcelona }\end{array}$ & icmab.es & 245 & 386 & 20 & 14 & 143 \\
\hline $\begin{array}{l}\text { Centro de Física Miguel A. } \\
\text { Catalán }\end{array}$ & cfmac.csic.es & 316 & 694 & 0 & 5 & 1 \\
\hline $\begin{array}{l}\text { Instituto de Ciencias de la } \\
\text { Tierra Jaume Almera }\end{array}$ & ija.csic.es & 885 & 1004 & 2 & 0 & 891 \\
\hline $\begin{array}{l}\text { Instituto de Robótica e } \\
\text { Informática }\end{array}$ & $\begin{array}{l}\text { www- } \\
\text { iri.upc.es }\end{array}$ & 268 & 414 & 0 & 0 & 77 \\
\hline
\end{tabular}




\section{Análisis de la presencia institucional del CSIC en el Web}

TABLA 11 (CONTINUACIÓN)

\begin{tabular}{|c|c|c|c|c|c|c|}
\hline $\begin{array}{l}\text { Centro de Estudios } \\
\text { Avanzados de Blanes }\end{array}$ & ceab.csic.es & 255 & 404 & 1 & 0 & 18 \\
\hline $\begin{array}{l}\text { Instituto de Física } \\
\text { Corpuscular }\end{array}$ & ific.uv.es & 1699 & 2406 & 15 & 1 & 53 \\
\hline $\begin{array}{l}\text { Instituto de Física de } \\
\text { Cantabria }\end{array}$ & ifca.unican.es & 752 & 962 & 1 & 0 & 0 \\
\hline $\begin{array}{l}\text { Instituto de Ciencia de } \\
\text { Materiales de Madrid }\end{array}$ & icmm.csic.es & 1537 & 1847 & 2 & 4 & 0 \\
\hline Instituto de la Grasa & ig.csic.es & 892 & 1016 & 0 & 0 & 0 \\
\hline $\begin{array}{l}\text { Centro de Investigación y } \\
\text { Desarrollo }\end{array}$ & cid.csic.es & 597 & 548 & 0 & 0 & 23 \\
\hline $\begin{array}{l}\text { Instituto de Astrofísica de } \\
\text { Andalucía }\end{array}$ & iaa.es & 1149 & 1014 & 0 & 1 & 0 \\
\hline $\begin{array}{l}\text { Centro Nacional de } \\
\text { Biotecnología }\end{array}$ & cnb.uam.es & 2455 & 1917 & 3 & 1 & 2 \\
\hline $\begin{array}{l}\text { Centro de Biología } \\
\text { Molecular Severo Ochoa }\end{array}$ & cbm.uam.es & 1818 & -1314 & 1 & 0 & 1 \\
\hline $\begin{array}{l}\text { Estación Biológica de } \\
\text { Doñana }\end{array}$ & ebd.csic.es & 4309 & 3062 & 7 & 0 & 0 \\
\hline Instituto de Acústica & \begin{tabular}{|l|} 
ia.csic.es \\
\end{tabular} & 1692 & 1172 & 1 & 0 & 1 \\
\hline $\begin{array}{l}\text { Instituto de Investigaciones } \\
\text { Biomédicas Alberto Sols }\end{array}$ & iib.uam.es & 2254 & 1279 & 0 & 0 & 0 \\
\hline $\begin{array}{l}\text { Instituto de Recursos } \\
\text { Naturales y Agrobiología de } \\
\text { Sevilla }\end{array}$ & irnase.csic.es & 1473 & 788 & 0 & 0 & 2 \\
\hline
\end{tabular}

\section{Ficheros Ricos}

El porcentaje de ficheros ricos que aparecen en las páginas Web de los distintos centros del CSIC, varía entre un 1,36 \% en el área de Ciencia y Tecnología Químicas y un 22,77 \% en el área de Ciencia y Tecnología Físicas. La composición de estos ficheros ricos se puede observar en la tabla 12. Los ficheros pdf aparecen en todas las áreas y ocupan entre un $39,9 \%$ y un $89,51 \%$ de los ficheros ricos. La abundancia de este tipo de ficheros en todas las áreas, se debe principalmente a que este tipo de formato se utiliza tanto en documentos burocráticos y administrativos, como en documentos de difusión científica. Los ficheros con formato rtfo $d o c$, también se utilizan para difusión científica, y se encuentran en todas las áreas del CSIC. Son muy abundantes en el área de Ciencia y Tecnología de Alimentos (41,89\%), lo que probablemente se debe a los artículos de divulgación con interés social. En el área de Humanidades y Ciencias Sociales los ficheros con formato doc representan un 30,03 \% de los ficheros ricos, debido probablemente a los textos de divulgación, libros, revistas, de los centros de esta área. 
TABLA 12. Tipos de ficheros ricos

\begin{tabular}{|c|c|c|c|c|c|c|c|}
\hline AREA CIENTÍFICA & $\begin{array}{c}\text { \% FICHEROS } \\
\text { RICOS }\end{array}$ & pdf & ps/eps & rtf/doc & tex & ppt & xls \\
\hline Humanidades y CC Sociales & $16,78 \%$ & $69,96 \%$ & & $30,03 \%$ & & & \\
\hline Biología y Biomedicina & $11,22 \%$ & $70,57 \%$ & $9,44 \%$ & $12,64 \%$ & & $6,74 \%$ & $0,59 \%$ \\
\hline Recursos Naturales & $3,9 \%$ & $64,92 \%$ & $14,95 \%$ & $11,88 \%$ & $3,47 \%$ & $1,41 \%$ & $3,34 \%$ \\
\hline $\begin{array}{c}\text { CC Agrarias } \\
\text { Ciencia y Tecnologías } \\
\text { Físicas }\end{array}$ & $11,26 \%$ & $89,51 \%$ & & $7,69 \%$ & & $2,79 \%$ & \\
\hline $\begin{array}{c}\text { Ciencia y Tecnología de } \\
\text { Materiales }\end{array}$ & $22,77 \%$ & $87,42 \%$ & $3,05 \%$ & $9,51 \%$ & & & $0,25 \%$ \\
\hline $\begin{array}{c}\text { Ciencia y Tecnología de } \\
\text { Alimentos }\end{array}$ & $2,80 \%$ & $39,29 \%$ & & $41,89 \%$ & & & $18,80 \%$ \\
\hline $\begin{array}{c}\text { Ciencia y Tecnologías } \\
\text { Químicas }\end{array}$ & $6,2 \%$ & $72,41 \%$ & & $26,93 \%$ & $0,64 \%$ & & \\
\hline
\end{tabular}


Los ficheros ps/eps, tex, ppt y xls son menos abundantes y no están representados en todas las áreas. Los ficheros ps/eps se utilizan, dada su facilidad, para fórmulas, lo que explica su abundancia en el área de Ciencia y Tecnología Físicas. Llama la atención el 18,80 \% de ficheros con formato $x l s$ en el área de Ciencia y Tecnología de Alimentos, todos ellos se encuentran en el Web de Instituto de Investigaciones Marinas de esta área y se deben a las tablas correspondientes a los muestreos de pesquerías de las distintas zonas.

\section{Visibilidad del Web de las áreas Científicas del CSIC}

Además del tamaño, otro criterio para analizar el impacto del Web es la visibilidad del mismo, que refleja el número total de enlaces externos recibidos por un sitio Web. Hemos analizado la visibilidad en el Web de los distintos centros del CSIC correspondientes a las ocho áreas científico-técnicas. Como se observa en la Figura 3, las áreas de Biología y Biomedicina, Recursos Naturales y Ciencia y Tecnología Físicas, son las áreas con mayor visibilidad. Estas 3 áreas también eran las de mayor tamaño del Web lo que en parte es debido al mayor número de centros de estas áreas frente a otras. Ciencias Agrarias y Ciencia y Tecnología de Alimentos, por el contrario, son las áreas que reciben menos enlaces externos coincidiendo también en este caso con las que menor tamaño del Web tienen.

FigurA 4

\section{VSIBIIDAD DEL WEB}

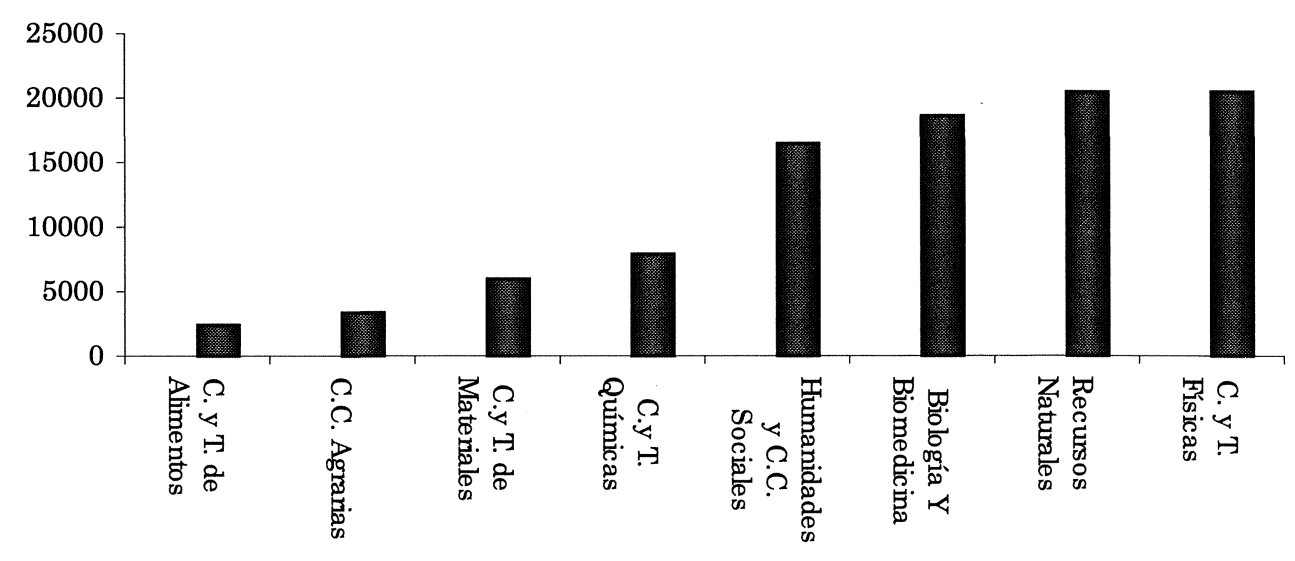


Isidro Aguillo Caño y Begoña Granadino Goenechea

TABLA 13

\begin{tabular}{|c|c|}
\hline AREA CIENTÍFICA & $\begin{array}{c}\text { VISIBILIDAD } \\
\text { (Alltheweb) }\end{array}$ \\
\hline Ciencia y Tecnología de Alimentos & 2.373 \\
\hline Ciencias Agrarias & 3.310 \\
\hline Ciencia y Tecnología de Materiales & 5.932 \\
\hline Ciencia y Tecnologías Químicas & 7.860 \\
\hline Humanidades y CC Sociales & 16.439 \\
\hline Biología y Biomedicina & 18.576 \\
\hline Recursos Naturales & 20.410 \\
\hline Ciencia y Tecnologías Físicas & 20.456 \\
\hline
\end{tabular}

En la tabla 14 se muestra la composición de la visibilidad; dominios gTLD, EU y IB. Entre los dominios gTLD, el dominio com es el mayoritario, entre los dominios europeos, el dominio es como era de esperar es el que aparece mas veces. Los dominios iberoamericanos suponen un porcentaje muy pequeño de la visibilidad. 
TABLA 14. Composición de la Visibilidad

\begin{tabular}{|c|c|c|c|c|c|c|c|c|}
\hline AREA CIENTÍfICA & com & org & net & edu & gov & es & $\begin{array}{c}\text { Resto EU } \\
\text { (fr/pt/nl/be/de } \\
\text { /uk/eu.int/it) }\end{array}$ & $\begin{array}{c}\text { IB } \\
(\mathrm{ar} / \mathrm{mx} / \mathrm{br} / \mathrm{co} / \\
\mathrm{cl} / \mathrm{ve} / \mathrm{uy} / \mathrm{pe})\end{array}$ \\
\hline Humanidades y Ciencias Sociales & $6,2 \%$ & $2,49 \%$ & $1,27 \%$ & $2,15 \%$ & $0 \%$ & $23,41 \%$ & $3,71 \%$ & $1,63 \%$ \\
\hline Biología y Biomedicina & $5,01 \%$ & $3,15 \%$ & $1,50 \%$ & $1,52 \%$ & $0,1 \%$ & $33,9 \%$ & $3,53 \%$ & $1,06 \%$ \\
\hline Recursos Naturales & $10,59 \%$ & $7,26 \%$ & $2,43 \%$ & $1,27 \%$ & $0,12 \%$ & $36,47 \%$ & $7,06 \%$ & $1,08 \%$ \\
\hline Ciencias Agrarias & $13,4 \%$ & $6,97 \%$ & $4,53 \%$ & $2,08 \%$ & $0,15 \%$ & $51,51 \%$ & $4,59 \%$ & $1,93 \%$ \\
\hline Ciencia y Tecnologías Físicas & $6,76 \%$ & $3,24 \%$ & $1,69 \%$ & $1,82 \%$ & $0,19 \%$ & $54,62 \%$ & $8,21 \%$ & $1,31 \%$ \\
\hline Ciencia y Tecnología de Materiales & $6,5 \%$ & $2,56 \%$ & $1,34 \%$ & $1,28 \%$ & $0,15 \%$ & $52 \%$ & $4,07 \%$ & $1,5 \%$ \\
\hline Ciencia y Tecnología de Alimentos & $15,76 \%$ & $6,32 \%$ & $3,45 \%$ & $1,05 \%$ & $0,25 \%$ & $73,49 \%$ & $4,80 \%$ & $1,85 \%$ \\
\hline Ciencia y Tecnologías Químicas & $5,66 \%$ & $2,45 \%$ & $1,67 \%$ & $0,69 \%$ & $0,05 \%$ & $24,79 \%$ & $4,40 \%$ & $1,22 \%$ \\
\hline
\end{tabular}

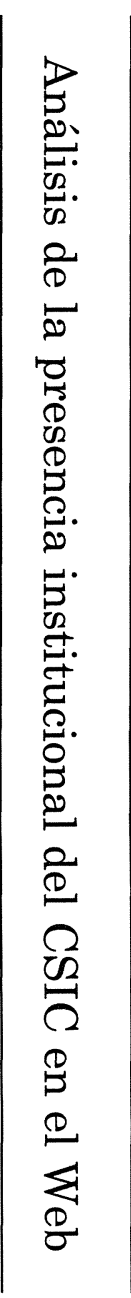

ํํํ 


\section{Visibilidad del Web por centros}

En el área de Humanidades y Ciencias Sociales, el CINDOC es el centro que recibe mayor número de enlaces externos, y contribuye con un $66,37 \%$ a la visibilidad total de esta área.

\section{TABLA 15. Humanidades y Ciencias Sociales}

\begin{tabular}{|l|c|c|}
\hline \multicolumn{1}{|c|}{ CENTRO DEL CSIC } & DOMINIO & VISIBILIDAD \\
\hline $\begin{array}{l}\text { Instituto de Estudios Islámicos y de Oriente } \\
\text { Próximo }\end{array}$ & ieiop.com & 8 \\
\hline Instituto de Arqueología de Mérida & iam.csic.es & 11 \\
\hline Instituto Histórico Hoffmeyer (HH) & ih.csic.es/presentacion/hoffmeyer & 35 \\
\hline Instituto de la Lengua Española & ile.csic.es & 42 \\
\hline Escuela de Estudios Árabes & iegps.csic.es & 59 \\
\hline $\begin{array}{l}\text { Instituto de Estudios Gallegos Padre } \\
\text { Sarmiento (IEGPS) }\end{array}$ & iesaa.csic.es & 64 \\
\hline $\begin{array}{l}\text { Instituto de Estudios Sociales de Andalucía } \\
\text { (IESA) }\end{array}$ & eeha.csic.es & 70 \\
\hline Escuela de estudios Hispano-Americanos & csic.it & 85 \\
\hline $\begin{array}{l}\text { Escuela Española de Historia y Arqueología } \\
\text { en Roma (EEHAR) }\end{array}$ & ingenio.upv.es & 109 \\
\hline $\begin{array}{l}\text { Instituto de la Gestión de la Innovación y del } \\
\text { Conocimiento (INGENIO) }\end{array}$ & iae-csic.uab.es & 128 \\
\hline Instituto de Análisis Económico & uv.es/ fresquet/TEXTOS & 183 \\
\hline $\begin{array}{l}\text { Instituto de Historia de la Ciencia y } \\
\text { Documentación Lopez Piñero }\end{array}$ & imf.csic.es & 10.911 \\
\hline Institución Milá y Fontanals & ieg.csic.es & 290 \\
\hline Instituto de Economía y Geografía & ceh.csic.es & 585 \\
\hline Instituto de Historia & filol.csic.es & 688 \\
\hline Instituto de Filología & ifs.csic.es & 739 \\
\hline Instituto de Filosofía (IFS) & cindoc.csic.es & 988 \\
\hline Unidad de Políticas Comparadas (UPC) & & 1428 \\
\hline $\begin{array}{l}\text { Centro de Información y Documentación } \\
\text { Científica (CINDOC) }\end{array}$ & & \\
\hline
\end{tabular}

En el área de Biología y Biomedicina, el CNB y el CBM son los centros con mayor número de enlaces y contribuyen a la visibilidad de esta área con un $44,45 \%$ y $20,89 \%$ respectivamente 


\section{Análisis de la presencia institucional del CSIC en el Web}

TABLA 16. Biología y Biomedicina

\begin{tabular}{|c|c|c|}
\hline CENTRO DEL CSIC & DOMINIO & VISIBILIDAD \\
\hline $\begin{array}{l}\text { Instituto de Biología y Genética Molecular, } \\
\text { Valladolid }\end{array}$ & ibgmva.csic.es & 6 \\
\hline Instituto de Bioquímica (IB) & ib.csic.es & 11 \\
\hline Unidad de Biofísica & ehu.es/biofisica & 11 \\
\hline $\begin{array}{l}\text { Instituto de Biología Molecular y Celular del } \\
\text { Cáncer (IBMCC) }\end{array}$ & www-cicancer.usal.es & 19 \\
\hline $\begin{array}{l}\text { Instituto de Farmacología y Toxicología } \\
\text { (IFT) }\end{array}$ & ift.csic.es & 22 \\
\hline $\begin{array}{l}\text { Instituto de Bioquímica Vegetal y } \\
\text { Fotosíntesis (IBVF) }\end{array}$ & ibvf.cartuja.csic.es & 48 \\
\hline Instituto de Biomedicina de Valencia (IBV) & ibv.csic.es & 67 \\
\hline $\begin{array}{l}\text { Instituto de Parasitología Y Biomedicina } \\
\text { López Neyra }\end{array}$ & ipb.csic.es & 68 \\
\hline Instituto de Neurociencias (IN) & in.umh.es & 69 \\
\hline Instituto de Microbiología Bioquímica & imb.usal.es & 106 \\
\hline $\begin{array}{l}\text { Instituto de Investigaciones Biomédicas de } \\
\text { Barcelona (IIBB) }\end{array}$ & iibb.csic.es & 135 \\
\hline $\begin{array}{l}\text { Instituto de Biología Molecular y Celular de } \\
\text { plantas'E Primo Yufera' }\end{array}$ & ibmcp.upv.es & 148 \\
\hline Instituto de Biología Molecular de Barcelona & ibmb.csic.es & 168 \\
\hline Instituto de Neurobiología Ramón y Cajal & cajal.csic.es & 491 \\
\hline $\begin{array}{l}\text { Instituto de Investigaciones Biomédicas } \\
\text { Alberto Sols (IIB) }\end{array}$ & iib.uam.es & 894 \\
\hline Centro de Investigaciones Biológicas (CIB) & cib.csic.es & 910 \\
\hline Centro de Investigación y Desarrollo (CID) & cid.csic.es & 1.107 \\
\hline $\begin{array}{l}\text { Centro de Investigaciones Científicas Isla de } \\
\text { la Cartuja (CIC) }\end{array}$ & cartuja.csic.es & 2.529 \\
\hline $\begin{array}{l}\text { Centro de Biología Molecular Severo Ochoa } \\
\text { (CBM) }\end{array}$ & cbm.uam.es & 3.881 \\
\hline Centro Nacional de Biotecnología & cnb.uam.es & 7.886 \\
\hline
\end{tabular}

En el área de Recursos Naturales el Museo Nacional de Ciencias Naturales es el centro con mayor visibilidad, como era de esperar y su contribución a la visibilidad del área es de un 48,01\%.

En Ciencias Agrarias, la Estación Experimental del Zaidín, contribuye con un $32,17 \%$. 


\section{Isidro Aguillo Caño y Begoña Granadino Goenechea}

TABLA 17. Recursos Naturales

\begin{tabular}{|c|c|c|}
\hline CENTRO DEL CSIC & DOMINIO & VISIBILIDAD \\
\hline Instituto Botánico de Barcelona & institutbotanic.bcn.es & 28 \\
\hline Instituto Andaluz de Ciencias de la Tierra (IACT) & ugr.es/ offiact/ & 38 \\
\hline $\begin{array}{l}\text { Instituto de Investigación en Recursos Cinegéticos } \\
\text { (IREC) }\end{array}$ & uclm.es/irec & 39 \\
\hline Instituto de Geología Económica (IGE) & ige.csic.es & 44 \\
\hline $\begin{array}{c}\text { Instituto de Recursos Naturales y Agrobiología de } \\
\text { Salamanca (IRNASA) }\end{array}$ & irnasa.csic.es & 46 \\
\hline Instituto de Astronomía y Geodesia (IAG) & mat.ucm.es/deptos/iag & 67 \\
\hline Instituto de Productos Naturales y Agrobiología (IPNA) & ipna.csic.es & 67 \\
\hline Centro de Investigación sobre desertificación & uv.es/cide/ & 92 \\
\hline Instituto de Acuicultura de Torre de la Sal & iats.csic.es & 105 \\
\hline Instituto de Ciencias Marinas de Andalucía (ICMAN) & icman.csic.es & 126 \\
\hline Unidad de Tecnología Marina (UTM) & utm.csic.es & 128 \\
\hline Instituto Pirenaico de Ecología (IPE) & ipe.csic.es & 167 \\
\hline Centro de Estudios Avanzados de Blanes (CEAB) & ceab.csic.es & 231 \\
\hline Estación Experimental de Zonas Áridas (EEZA) & eeza.csic.es & 297 \\
\hline Estación Biológica de Doñana & ebd.csic.es & 308 \\
\hline $\begin{array}{c}\text { Centro de Edafología y Biología aplicada del Segura } \\
\text { (CEBAS) }\end{array}$ & cebas.csic.es & 316 \\
\hline Centro de Ciencias medioambientales (CCMA) & ccma.csic.es & 327 \\
\hline $\begin{array}{l}\text { Instituto de Recursos Naturales y Agrobiología de Sevilla } \\
\text { (IRNAS) }\end{array}$ & irnase.csic.es & 332 \\
\hline Real Jardín Botánico (RJB) & rjb.csic.es & 473 \\
\hline Instituto de Agroquímica y Tecnología de los Alimentos & iata.csic.es & 765 \\
\hline $\begin{array}{c}\text { Centro Mediterráneo de investigaciones marinas y } \\
\text { medioambientales (UTM+ ICM) }\end{array}$ & cmima.csic.es & 870 \\
\hline Instituto de Investigaciones Marinas & iim.csic.es & 988 \\
\hline $\begin{array}{l}\text { Instituto Medfiterráneo de Estudios Avanzados } \\
\text { (IMEDEA) }\end{array}$ & imedea.uib.es & 998 \\
\hline Estación Experimental del Zaidín & eez.csic.es & 1.065 \\
\hline Instituto de Ciencias de la Tierra Jaume Almera & ija.csic.es & 1.317 \\
\hline Instituto de Ciencias del Mar & icm.csic.es & 1.376 \\
\hline Museo Nacional de Ciencias Naturales (MNCN) & mncn.csic.es & 9.800 \\
\hline
\end{tabular}




\section{Análisis de la presencia institucional del CSIC en el WEB}

TABLA 18. Ciencias Agrarias

\begin{tabular}{|c|c|c|}
\hline $\begin{array}{c}\text { CENTRO DEL CSIC } \\
\end{array}$ & DOMINIO & VISIBILIDAD \\
\hline $\begin{array}{l}\text { Instituto de Agrobiotecnología y Recursos } \\
\text { Naturales (IARN), Navarra }\end{array}$ & unavarra.es/invest/biotec.htm & 7 \\
\hline Estación Agrícola Experimental (EAE), León & eae.csic.es & 22 \\
\hline $\begin{array}{l}\text { Instituto de Investigaciones Agrobiológicas de } \\
\text { Galicia (IIAG) }\end{array}$ & iiag.csic.es & 34 \\
\hline Instituto de Agricultura Sostenible (IAS), Córdoba & ias.csic.es & 39 \\
\hline $\begin{array}{l}\text { Instituto de Recursos Naturales y Agrobiología de } \\
\text { Salamanca (IRNASA) }\end{array}$ & irnasa.csic.es & 46 \\
\hline $\begin{array}{l}\text { Estación Experimental La Mayora (EELM), } \\
\text { Málaga }\end{array}$ & eelm.csic.es & 47 \\
\hline Misión Biológica de Galicia (MBG) & mbg.csic.es & 51 \\
\hline $\begin{array}{l}\text { Instituto de Productos Naturales y Agrobiología } \\
\text { (IPNA), Tenerife }\end{array}$ & ipna.csic.es & 67 \\
\hline $\begin{array}{l}\text { Estación Experimental de Aula Dei (EEAD), } \\
\text { Zaragoza }\end{array}$ & eead.csic.es & 192 \\
\hline $\begin{array}{l}\text { Centro de Edafología y Biología Aplicada del } \\
\text { Segura (CEBAS), Murcia }\end{array}$ & cebas.csic.es & 316 \\
\hline $\begin{array}{l}\text { Centro de Ciencias Medioambientales (CCMA), } \\
\text { Madrid }\end{array}$ & ccma.csic.es & 327 \\
\hline $\begin{array}{l}\text { Instituto de recursos Naturales y Agrobiología de } \\
\text { Sevilla (IRNAS) }\end{array}$ & irnase.csic.es & 332 \\
\hline $\begin{array}{l}\text { Instituto de Agroquímica y Tecnología de } \\
\text { Alimentos (IATA), Valencia }\end{array}$ & iata.csic.es & 765 \\
\hline Estación Experimental del Zaidín (EEZ), Granada & eez.csic.es & 1.065 \\
\hline
\end{tabular}

En Ciencia y Tecnologías Físicas, el Instituto de Investigación en Inteligencia Artificial es el de mayor visibilidad con una contribución del $37,43 \%$. El IFIC a pesar de tener un alto número de páginas web, recibe pocos enlaces externos y tiene por tanto una visibilidad baja. 


\section{Isidro Aguillo Caño y Begoña Granadino Goenechea}

Tabla 19. Ciencia y Tecnologías Físicas

\begin{tabular}{|l|c|c|}
\hline \multicolumn{1}{|c|}{ CENTRO DEL CSIC } & DOMINIO & VISIBILIDAD \\
\hline Centro Nacional de Aceleradores (CNA) & us.es/cna & 7 \\
\hline Observatorio de Física Cósmica del Ebro (OE) & obsebre.es & 21 \\
\hline $\begin{array}{l}\text { Laboratorio de Física de Sistemas Pequeños y } \\
\text { Nanotecnología }\end{array}$ & fsp.csic.es & 62 \\
\hline Instituto de Astronomía y Geodesia (IAG) & mat.ucm.es/deptos/iag & 67 \\
\hline Instituto de Öptica Daza de Valdés & io.cfmac.csic.es & 73 \\
\hline Instituto de Física Aplicada (IFA) & ifa.csic.es & 88 \\
\hline $\begin{array}{l}\text { Instituto de Microelectrónica de Barcelona (IMB- } \\
\text { CNM) }\end{array}$ & cnm.es/imb & 94 \\
\hline Instituto de Estructura de la Materia (IEM) & iem.cfmac.csic.es & 100 \\
\hline Instituto de Robótica e Informática (IRII) & www-iri.upc.es & 172 \\
\hline Instituto de Microelectrónica de Madrid & imm.cnm.csic.es & 173 \\
\hline Instituto de Física Teórica & gesalerico.ft.uam.es & 233 \\
\hline $\begin{array}{l}\text { Instituto de Matemática y Física Fundamental } \\
\text { (IMAFF) }\end{array}$ & imaff.cfmac.csic.es & $170+83$ \\
\hline Instituto de Microelectrónica de Sevilla & imse.cnm.es & 304 \\
\hline Instituto de Automática Industrial & iai.csic.es & 330 \\
\hline Instituto de Acústica & ia.csic.es & 557 \\
\hline Centro de Astrobiología (CAB) & cab.inta.es & 662 \\
\hline Instituto de Estudios Espaciales de Cataluña & ieec.fr.es & 668 \\
\hline Instituto de Física de Cantabria (IFCA) & ifca.unican.es & 933 \\
\hline Centro Técnico de Informática (CTI) & cti.csic.es & 969 \\
\hline $\begin{array}{l}\text { Instituto Mediterráneo de Estudios Avanzados } \\
\text { (IMEDEA) }\end{array}$ & www-iri.upc.es & 998 \\
\hline Instituto de Astrofísica de Andalucía & iaa.es & 1017 \\
\hline Instituto de Física Corpuscular (IFIC) & ific.uv.es & 1.435 \\
\hline $\begin{array}{l}\text { Instituto de Investigación en Inteligencia } \\
\text { artificial (IIIA) }\end{array}$ & iiia.csic.es & 7.657 \\
\hline
\end{tabular}

\begin{tabular}{|l|c|c|}
\hline SUPERSEDES & & \\
\hline Centro de Física Miguel A. Catalán (CFMAC) & cfmac.csic.es & 671 \\
\hline $\begin{array}{l}\text { Centro de Tecnologías Físicas Leonardo Torres } \\
\text { Quevedo (CETEF) }\end{array}$ & cetef.csic.es & 1.224 \\
\hline Centro nacional de Microelectrónica (CNM) & cnm.es & 1.670 \\
\hline
\end{tabular}

En Tecnología de Alimentos, El Instituto de Investigaciones Marinas y el Instituto de Agroquímica y Tecnología de Alimentos contribuyen a la visibilidad del área con un $42,63 \%$ y $32,23 \%$ respectivamente. 


\section{Análisis de la presencia institucional del CSIC en el Web}

TABLA 20. Tecnología de Alimentos

\begin{tabular}{|l|c|c|}
\hline \multicolumn{1}{|c|}{ CENTRO DEL CSIC } & DOMINIO & VISIBILIDAD \\
\hline $\begin{array}{l}\text { Instituto de Nutrición y Bromatología } \\
\text { (INB) }\end{array}$ & & 25 \\
\hline $\begin{array}{l}\text { Instituto de Productos Lácteos de } \\
\text { Asturias (IPLA) }\end{array}$ & ipla.csic.es & 42 \\
\hline $\begin{array}{l}\text { Instituto de Fermentaciones } \\
\text { Industriales (IFI) }\end{array}$ & ifi.csic.es & 91 \\
\hline Instituto del Frío (IF) & if.csic.es & 146 \\
\hline Instituto de La Grasa (IG) & ig.csic.es & 316 \\
\hline $\begin{array}{l}\text { Centro de Edafología Y Biología } \\
\text { Aplicada del Segura (CEBAS) }\end{array}$ & cebas.csic.es & 765 \\
\hline $\begin{array}{l}\text { Instituto de Agroquímica y Tecnología } \\
\text { de Alimentos (IATA) }\end{array}$ & iata.csic.es & 988 \\
\hline $\begin{array}{l}\text { Instituto de Investigaciones Marinas } \\
\text { (IIM) }\end{array}$ & iim.csic.es & \\
\hline
\end{tabular}

En Tecnología de Materiales hay 3 centros con una visibilidad mayor que los demás: El ICMAB, el ICMM y el CIC y contribuyen con un $18,5 \%$, $20,58 \%$ y $42,63 \%$ respectivamente.

TABLA 21. Tecnología de Materiales

\begin{tabular}{|l|c|c|}
\hline \multicolumn{1}{|c|}{ CENTRO DEL CSIC } & DOMINIO & VISIBILIDAD \\
\hline $\begin{array}{l}\text { Instituto de Ciencias Materiales de Sevilla } \\
\text { (ICMS) }\end{array}$ & icmse.cartuja.csic.es & 46 \\
\hline $\begin{array}{l}\text { Instituto de Ciencias Materiales de Aragón } \\
\text { (ICMA) }\end{array}$ & icma.csic.unizar.es & 56 \\
\hline Instituto de Cerámica y vidrio (ICV) & icv.csic.es & 107 \\
\hline $\begin{array}{l}\text { Centro Nacional de Investigaciones Metalúrgicas } \\
\text { (CENIM) }\end{array}$ & cenim.csic.es & 212 \\
\hline $\begin{array}{l}\text { Instituto de Ciencias de la Construcción Eduardo } \\
\text { Torroja (IETCC) }\end{array}$ & ietcc.csic.es \\
\hline $\begin{array}{l}\text { Instituto de Ciencia y Tecnología de polímeros } \\
\text { (ICTP) }\end{array}$ & ictp.csic.es & 215 \\
\hline Unidad de Física de Materiales (UFM) & csic.sw.ehu.es & 272 \\
\hline $\begin{array}{l}\text { Instituto de Ciencias Materiales de Barcelona } \\
\text { (ICMAB) }\end{array}$ & icmab.es & $7+1.091$ \\
\hline $\begin{array}{l}\text { Instituto de Ciencias Materiales de Madrid } \\
\text { (ICMM) }\end{array}$ & icmm.csic.es & \multirow{2}{1}{221} \\
\hline $\begin{array}{l}\text { Centro de Investigaciones Científicas Isla } \\
\text { de la Cartuja (CIC) }\end{array}$ & cartuja.csic.es & 2.529 \\
\hline
\end{tabular}

En el área de Ciencia y Tecnologías Químicas destacan el IIQ con un $31,42 \%$ y el CIC con un $32,17 \%$. Llama la atención el hecho de que el Instituto de Química Física Rocasolano tenga una visibilidad baja, a pesar de ser el segundo centro del CSIC con mayor tamaño del Web. 


\section{Isidro Aguillo Caño y Begoña Granadino Goenechea}

TABLA 22. Ciencia y Tecnologías Quimicas

\begin{tabular}{|l|c|c|}
\hline \multicolumn{1}{|c|}{ CENTRO DEL CSIC } & DOMINIO & VISIBILIDAD \\
\hline Instituto de Química Médica (IQM) & iqm.csic.es & 41 \\
\hline $\begin{array}{l}\text { Instituto de Ciencia de Mațeriales de } \\
\text { Aragón (ICMA) }\end{array}$ & icma.csic.unizar.es & 56 \\
\hline $\begin{array}{l}\text { Instituto de Productos Naturales y } \\
\text { Agrobiología (IPNA) }\end{array}$ & ipna.csic.es & 67 \\
\hline Instituto de Carboquímica & icb.csic.es & 81 \\
\hline $\begin{array}{l}\text { Laboratorio de Investigación en } \\
\text { Tecnologías de la Combustión (LITEC) }\end{array}$ & litec.csic.es & 90 \\
\hline $\begin{array}{l}\text { Instituto de Química Orgánica General } \\
\text { (IQOG) }\end{array}$ & iqo.csic.es & $91+47$ \\
\hline Instituto de Tecnología Química (ITQ) & upv.es/itq & 151 \\
\hline $\begin{array}{l}\text { Instituto de Investigaciones Químicas y } \\
\text { Ambientales J.P.Vila (IIQAB) }\end{array}$ & iiqab.csic.es \\
\hline Instituto Nacional del Carbón (INCAR) & incar.csic.es & 159 \\
\hline $\begin{array}{l}\text { Instituto de Química Física Rocasolano } \\
\text { (IQFR) }\end{array}$ & iqfr.csic.es & 209 \\
\hline Instituto de Catálisis y Petroleoquímica & icp.csic.es & 246 \\
\hline Centro de Investigación y Desarrollo & cid.csic.es & 515 \\
\hline $\begin{array}{l}\text { Instituto de Investigaciones Químicas } \\
\text { (IIQ) }\end{array}$ & iiq.cartuja.csic.es & 1.071 \\
\hline $\begin{array}{l}\text { Centro de Investigaciones Científicas } \\
\text { Isla de la Cartuja (CIC) }\end{array}$ & cartuja.csic.es & 2.470 \\
\hline
\end{tabular}

Con estos datos podemos concluir que los 4 centros del CSIC con mayor visibilidad son:

- El Centro de Información y documentación científica (CINDOC) del área de Humanidades y Ciencias Sociales.

- Museo Nacional de Ciencias Naturales (MNCN) del área de Recursos Naturales. 9.800

- Centro Nacional de Biotecnología (CNB) del área de Biología y Biomedicina 7.886

- Instituto de Investigaciones en Inteligencia Artificial (IIIA) del área de Ciencia y Tecnologías Físicas... 7.657

De estos 4 centros tanto el CNB como el MNCN representan también 2 de los 4 centros del CSIC de mayor tamaño del Web.

En la siguiente tabla mostramos un resumen del tamaño y de la visibilidad del Web de las 8 áreas del CSIC en relación al número de centros que forman cada una de estas áreas. 


\section{Análisis de la presencia institucional del CSIC en el Web}

TABLA 23

\begin{tabular}{|c|c|c|c|}
\hline AREA CIENTIFICA & TAMAÑO & VISIBILIDAD & N $^{\circ}$ DE CENTROS \\
\hline $\begin{array}{c}\text { Ciencia y Tecnología de } \\
\text { Alimentos }\end{array}$ & 4.092 & 2.373 & 8 \\
\hline $\begin{array}{c}\text { Ciencia y Tecnología de } \\
\text { Materiales }\end{array}$ & 7.583 & 5.932 & 10 \\
\hline Ciencias Agrarias & 8.514 & 3.310 & 14 \\
\hline Humanidades y CC Sociales & 11.136 & 16.439 & 19 \\
\hline $\begin{array}{c}\text { Ciencia y Tecnologías } \\
\text { Químicas }\end{array}$ & 34.046 & 7.860 & 14 \\
\hline Ciencia y Tecnologías Físicas & 34.783 & 20.456 & 28 \\
\hline Biología y Biomedicina & 42.332 & 18.576 & 20 \\
\hline Recursos Naturales & 50.429 & 20.410 & 27 \\
\hline
\end{tabular}

\section{Popularidad según Ranking de Alexa}

En la tabla 23 mostramos la popularidad según el Ranking Alexa del CSIC en el contexto de las 12 universidades españolas con mayor Ranking. Como se puede ver en la tabla, el CSIC aparece en la posición 6.857, con 5 universidades españolas con mayor popularidad.

TABLA 24

\begin{tabular}{|c|c|c|}
\hline Institución & Dirección & Posición \\
\hline Universidad abierta de Cataluña & uoc.edu/uoc.es & 2.070 \\
\hline $\begin{array}{c}\text { Universidad Nacional de Educación a } \\
\text { Distancia }\end{array}$ & uned.es & 3.517 \\
\hline Universidad Complutense de Madrid & ucm.es & 4.158 \\
\hline Universidad de Zaragoza & unizar.es & 5.546 \\
\hline Universidad de Alicante & ua.es & 5.739 \\
\hline CSIC & csic.es & 6.857 \\
\hline Universidad de Barcelona & ub.es & 7.044 \\
\hline Universidad de Sevilla & us.es & 7.089 \\
\hline Universidad de Oviedo & uniovi.es & 7.275 \\
\hline Universidad de Valencia & uv.es & 8.097 \\
\hline Universidad Politécnica de Madrid & upm.es & 8.310 \\
\hline Universidad Autónoma de Barcelona & uab.es & 9.537 \\
\hline Universidad Autónoma de Madrid & uam.es & 9.751 \\
\hline Universidad del País Vasco & ehu.es & 10.454 \\
\hline Universidad Carlos III & uc3m.es & 11.676 \\
\hline Universidad de Navarra & unav.es & 14.114 \\
\hline Universidad Politécnica de Valencia & upv.es & 14.171 \\
\hline Universidad Politécnica de Cataluña & upc.es & 14.259 \\
\hline Universidad de Cádiz & uca.es & 15.200 \\
\hline Universidad de Málaga & uma.es & 15.491 \\
\hline
\end{tabular}


138

\section{Isidro Aguillo Caño y Begoña Granadino Goenechea}

En la tabla 24 se muestra la contribución relativa de las sedes a la popularidad del CSIC. Como se ve en la tabla, la página Web principal del CSIC es la mas que contribuye a la popularidad del CSIC, seguida por la página del CINDOC, que también tiene una contribución alta.

TABLA 25. Contribución relativa de las sedes a la popularidad del CSIC

\begin{tabular}{|c|c|c|}
\hline Sede & Dirección & Porcentaje \\
\hline CSIC & csic.es & $29 \%$ \\
\hline $\begin{array}{c}\text { Centro de Información y Documentación } \\
\text { Científica }\end{array}$ & cindoc.csic.es & $23 \%$ \\
\hline Catálogo Bibliográfico del CSIC & aleph.csic.es/sauco.csic.es & $7 \%$ \\
\hline $\begin{array}{l}\text { Dpto. Tratamiento de la información. } \\
\text { Instituto de Física Aplicada }\end{array}$ & iec.csic.es & $3 \%$ \\
\hline Portal Mayores & imsersomayores.csic.es & $3 \%$ \\
\hline Residencia de Estudiantes & residencia.csic.es/luisbunuel.org & $3 \%$ \\
\hline Emumail-CTI & naranjo.csic.es & $2 \%$ \\
\hline Museo Nacional de Ciencias Naturales & mncn.csic.es & $2 \%$ \\
\hline Unidad de Tecnología Marina & utm.csic.es & $2 \%$ \\
\hline Bases de Datos del CSIC & bddoc.csic.es/bbdd.csic.es & $2 \%$ \\
\hline Instituto de Economía y Geografía & ieg.csic.es & $1 \%$ \\
\hline Instituto de Humanidades & ih.csic.es & $1 \%$ \\
\hline $\begin{array}{l}\text { Instituto de Recursos Naturales y } \\
\text { Agrobiología de Sevilla }\end{array}$ & irnase.csic.es & $1 \%$ \\
\hline Instituto de Filosofía & ifs.csic.es & $1 \%$ \\
\hline Real Jardín Botánico & rjb.csic.es & $1 \%$ \\
\hline Centro Técnico de Informática & cti.csic.es & $1 \%$ \\
\hline $\begin{array}{c}\text { Instituto de Ciencias de la Construcción } \\
\text { Eduardo Torroja }\end{array}$ & ietcc.csic.es & $1 \%$ \\
\hline Instituto de Catálisis y Petroleoquímica & icp.csic.es & $1 \%$ \\
\hline Instituto de Ciencias del Mar & icm.csic.es & $1 \%$ \\
\hline Instituto de Filología & filol.csic.es & $1 \%$ \\
\hline $\begin{array}{c}\text { Instituto de Ciencia de Materiales de } \\
\text { Madrid }\end{array}$ & icmm.csic.es & $1 \%$ \\
\hline Estación Experimental de Zonas Áridas & eeza.csic.es & $1 \%$ \\
\hline Centro de Investigaciones Biológicas & cib.csic.es & $1 \%$ \\
\hline Otras Sedes & & $11 \%$ \\
\hline
\end{tabular}

\section{Bibliografía}

Almind, T. C.; IngWersen, P., (1997): Informetric analyses on the World Wide Web: A methodological approach to "webometrics». Journal of Documentation, 53(4):404-426.

AguiLlo, I. F. (1998): STM Information on the Web and the development of new Internet R\&D databases and indicators. Proc. Online Meeting 1998. pp. 239-243. Learned Information, Londres.

AGuillo, I. F. (2000): Contenidos de I+D en Internet: Mitos y leyendas. Mundo Cientifico, 211:22-25.

AGUILlo, I. F. (2002): Measuring informal scientific communication in the Web. EASST 2002 Conference. University of York. 


\section{Análisis de la presencia institucional del CSIC en el Web}

BAR-ILAN, J. (2002). Methods for assessing search engine performance over time. Journal of the American Society for Information Science and Technology, 53(4):308-319.

BAR-ILAN, J. (2004): Search engine ability to cope with the changing web. Levene, Mark; Poulovassilis, Alexandra (Eds.)Web Dynamics Adapting to Change in Content, Size, Topology and Use. Springer Verlag.

BJORNEBORN, L. \& (2001): Perspectives of webometrics. Scientometrics, 50(1): 65-82.

BJORNEBORN, L. (2004): Small-world link structures across an academic web space : a library and information science approach. PhD dissertation. Royal School of Library and Information Science. xxxvi, 399 p. ISBN 87-7415-276-9

$<\mathrm{http} / / / \mathrm{www} \cdot \mathrm{db} . \mathrm{dk} / \mathrm{lb} / \mathrm{phd} / \mathrm{phd}$-thesis.pdf>

EICSTES Consortium (2002). Development of web indicators. <http://www.eicstes.org/ EICSTES PDF/Deliverables/Development\%20of\%20Web\%20Indicators.PDF>

INGWERSEN, P. (1998): The Calculation of Web Impact Factors. Journal of Documentation, 54(2): 236-243.

INGWERsEN, P. \& BJORNEBORN, L. (2004): Methodological issues of webometric studies. Glanzel, W. et al. (eds.). Quantitative Science and Technology Research. Kluwer Academic Publishers.

LARSON, R. A.(1996): Bibliometrics of the World Wide Web: An Exploratory Analysis of the Intellectual Structure of Cyberspace. Proceedings of ASIS 1996 Annual Meeting, Baltimore, October 19-24, 1996. pp. 71-78. $<$ http://sherlock.berkeley.edu/asis96/asis96.html>

Li, X., Thelwall, M., Musgrove, P. \& Wilkinson, D. (2003): The relationship between the links/Web Impact Factors of computer science departments in UK and their RAE (Research Assessment Exercise) ranking in 2001, Scientometrics 57(2), 239-255.

RousseaU, R. (1997): Sitations: an exploratory study. Cybermetrics, 1(1): paper 1. $<$ http://www.cindoc.csic.es/cybermetrics/articles/v1i1p1.html>.

SмiтH, A. G. (1999). A tale of two web spaces: comparing sites using web impact factors. Journal of Documentation, 55 (5):577-592.

Thelwall, M. \& Aguillo, I. F. (2003). La salud de las web universitarias españolas. Revista Española Documentación Científica, 26(3): 291-305.

Thelwall, M. \& HaRries, G. (2004): Journal of the American Society for Information Science and Technology, 55(2), 149-159.

THELWALL, M. \& HARRIES, G. (2003): An Investigation Based Upon a Classification of the Relationships of Pages to the Research of the Host University. Journal of the American Society for Information Science and Technology, 54(7), 594-602.

Thelwall, M. VAughan \& BJoRneborn, L. (2005): Webometrics. Annual Review of Information Science and Technology, 39.

VAUGHAN, L. \& Thelwall, M. (2003): Scholarly use of the Web: What are the key inducers of links to journal Web sites? Journal of the American Society for Information Science and Technology, 54(1), 29-38.

VAUGHAN, L.(2004): New measurements for search engine evaluation proposed and tested. Information Processing \& Management,40 (en prensa).

Vaughan, L. \& Thelwall, M. (2004). Search engine coverage bias: evidence and possible causes. Information Processing \& Management,40 (en prensa).

Wilkinson, D., Harries, G., Thelwall, M. \& Price, E. (2003): Motivations for academic Web site interlinking: Evidence for the Web as a novel source of information on informal scholarly communication, Journal of Information Science, 29(1), 29(1), 59-66. 